\title{
Toward the probabilistic forecasting of high-latitude GPS phase scintillation
}

\author{
P. Prikryl, ${ }^{1}$ P. T. Jayachandran, ${ }^{2}$ S. C. Mushini, ${ }^{2}$ and I. G. Richardson ${ }^{3,4}$
}

Received 9 April 2012; revised 20 June 2012; accepted 21 June 2012; published 14 August 2012.

[1] The phase scintillation index was obtained from L1 GPS data collected with the Canadian High Arctic Ionospheric Network (CHAIN) during years of extended solar minimum 2008-2010. Phase scintillation occurs predominantly on the dayside in the cusp and in the nightside auroral oval. We set forth a probabilistic forecast method of phase scintillation in the cusp based on the arrival time of either solar wind corotating interaction regions (CIRs) or interplanetary coronal mass ejections (ICMEs). CIRs on the leading edge of high-speed streams (HSS) from coronal holes are known to cause recurrent geomagnetic and ionospheric disturbances that can be forecast one or several solar rotations in advance. Superposed epoch analysis of phase scintillation occurrence showed a sharp increase in scintillation occurrence just after the arrival of high-speed solar wind and a peak associated with weak to moderate CMEs during the solar minimum. Cumulative probability distribution functions for the phase scintillation occurrence in the cusp are obtained from statistical data for days before and after CIR and ICME arrivals. The probability curves are also specified for low and high (below and above median) values of various solar wind plasma parameters. The initial results are used to demonstrate a forecasting technique on two example periods of CIRs and ICMEs.

Citation: Prikryl, P., P. T. Jayachandran, S. C. Mushini, and I. G. Richardson (2012), Toward the probabilistic forecasting of high-latitude GPS phase scintillation, Space Weather, 10, S08005, doi:10.1029/2012SW000800.

\section{Introduction}

[2] Interplanetary coronal mass ejections (ICMEs) and corotating interaction regions (CIRs) on the leading edge of high-speed streams (HSSs) from coronal holes are major causes of ionospheric disturbances. The strongest of these solar wind events result in major geomagnetic storms [Tsurutani and Gonzalez, 1997; Richardson et al., 2006; Tsurutani et al., 2006; Badruddin, 2009], which have a global impact on the ionosphere [Buonsanto, 1999; Mannucci et al., 2005; Pokhotelov et al., 2010; Pedatella and Forbes, 2011]. Although there are many differences between CIR and CME driven geomagnetic storms [Borovsky and Denton, 2006], they both result in enhanced ionospheric irregularities causing radio wave amplitude and phase scintillation,

\footnotetext{
${ }^{1}$ Communications Research Centre Canada, Ottawa, Ontario, Canada.

${ }^{2}$ Physics Department, University of New Brunswick, Fredericton, New Brunswick, Canada.

${ }^{3}$ CRESST and Department of Astronomy, University of Maryland, College Park, Maryland, USA.

${ }^{4}$ Also at NASA Goddard Space Flight Center, Greenbelt, Maryland, USA.
}

Corresponding author: P. Prikryl, Communications Research Centre Canada, Ottawa, ON K2H 8S2, Canada. (paul.prikryl@crc.gc.ca) which can affect operation of satellite communication and navigation systems [Aarons, 1982; Basu et al., 2002]. Scintillation at radio frequencies from VHF to L band has been intensely studied for geomagnetic storms at low latitudes [Crane, 1976; Dabas et al., 1989; Aarons, 1982; Basu et al., 2005; Ray and DasGupta, 2007; Li et al., 2006, 2008; de Abreu et al., 2010], and at high latitudes [Aarons, 1982, 1997; Aarons et al., 2000; Mitchell et al., 2005]. Ionospheric scintillation degrades GPS positioning accuracy and causes cycle slips, which can lead to complete loss of signal phase lock and operational outages. The impact on signal tracking performance of a GPS receiver was studied theoretically [Hegarty et al., 2001; Conker et al., 2003] and practically [Skone and de Jong, 2000; Sreeja et al., 2011; Strangeways et al., 2011].

[3] Ionospheric scintillation is difficult to predict for a given raypath because of complexity of ionospheric irregularity generation, coupling of the solar wind magnetic and electric fields to the ionosphere, and variable convection of ionospheric plasma. Past research and development of methods for specification and forecasting of scintillation have focused on the equatorial region where, due to the presence of an equatorial anomaly, the most intense scintillation is known to occur after sunset [Basu et al., 2002, 2010; Anderson et al., 2004; de La Beaujardière et al., 2004; Redmon et al., 2010]. However, statistical (empirical) models of scintillation have been developed for 


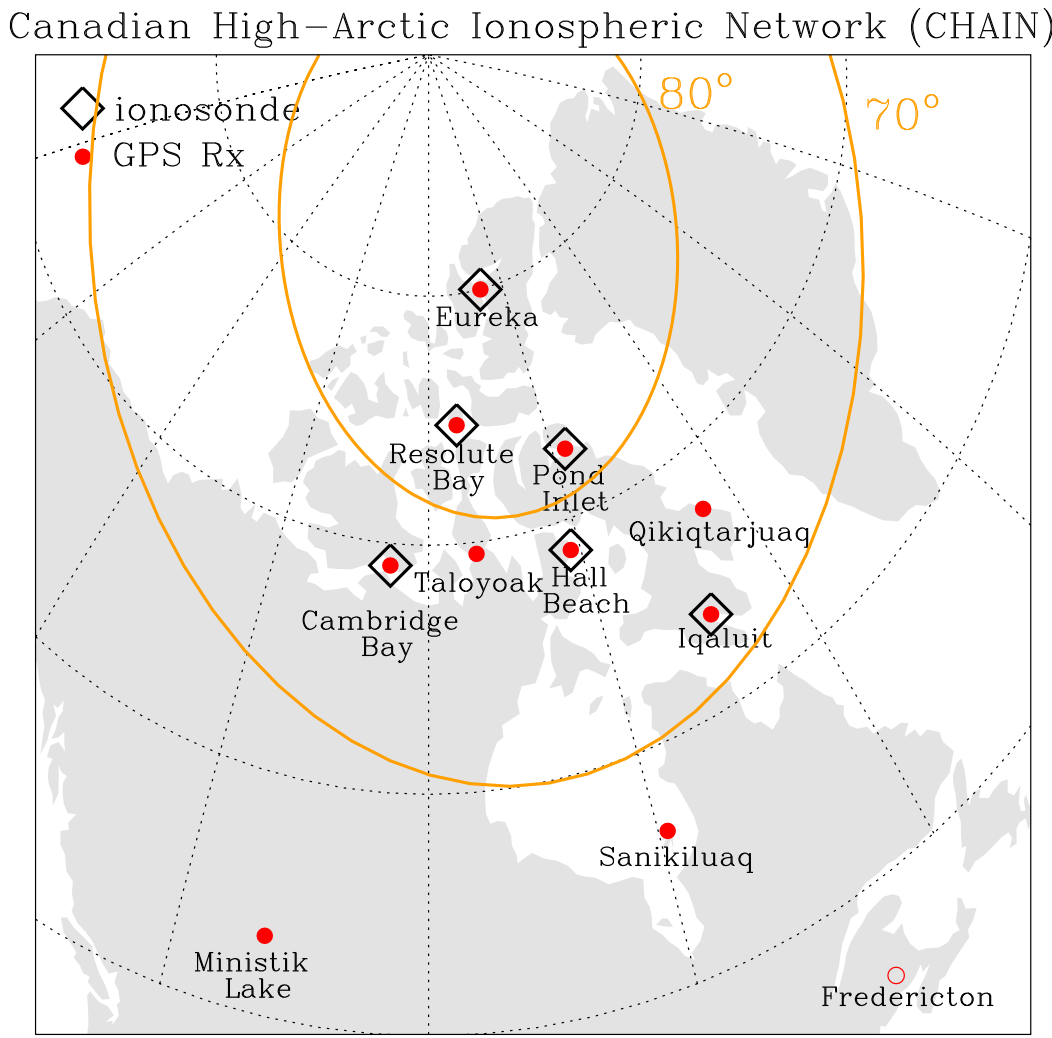

Figure 1. Canadian High Arctic Ionospheric Network (CHAIN). The corrected geomagnetic (CGM) latitudes $70^{\circ}$ and $80^{\circ}$ are superposed over the geographic grid.

both the equatorial and high-latitude ionospheres [Secan et al., 1995, 1997]. These models used scintillation data from past satellite experiments (Wideband, HiLat and Polar BEAR) that covered frequencies from VHF to L-band, although the modeling was based on VHF data only. More recently, using satellite in situ measurements of plasma (electron) density fluctuations, IRI ionosphere model, and the phase screen propagation model, a scintillation climatology model for the high-latitude ionosphere was developed [Wernik, et al., 2007]. As the number of ground-based scintillation monitors of global navigational satellite systems (GNSS) signals in the auroral and polar regions increases, it has become possible to construct scintillation climatology from accumulated scintillation data [Spogli et al., 2009; Prikryl et al., 2011a] and to address the questions of forecasting and mitigation of L-band scintillation at high-latitudes [Decker and Pedersen, 2001; Aquino et al., 2007; Strangeways et al., 2011]. In this paper we present observations of GPS phase scintillation following the arrivals of solar wind disturbances (ICMEs and HSS/CIRs) and discuss possibilities of forecasting the phase scintillation occurrence at high latitudes.

\section{Instruments and Data}

[4] The Canadian High Arctic Ionospheric Network (CHAIN) [Jayachandran et al., 2009] consists of ten specialized GPS receivers and six Canadian Advanced Digital Ionosondes (CADIs) distributed in the auroral oval, cusp and the polar cap (http://chain.physics.unb.ca/chain). A brief description of CHAIN instruments, the scintillation parameters obtained and the geographic and corrected geomagnetic coordinates of stations can be found elsewhere [e.g., Prikryl et al., 2011a].

[5] The CHAIN (Figure 1) is equipped with NovAtel OEM4 dual frequency GPS receivers GSV 4004B [Van Dierendonck and Arbesser-Rastburg, 2004] with special firmware specifically configured to measure and record power and phase of the GPS L1 signal at high sampling rate $(50 \mathrm{~Hz})$. The receiver computes ionospheric total electron content (TEC) using both GPS L1 and L2 signals, the amplitude scintillation index $S_{4}$ and the phase scintillation index $\sigma_{\Phi}$ that is used in this study. The phase scintillation index $\sigma_{\Phi}$ is the standard deviation of the detrended phase using a filter in the receiver with $0.1 \mathrm{~Hz}$ lower cutoff frequency. Intense scintillation is usually accompanied by cycle slips. A cycle slip [Horvath and Crozier, 2007] is defined here as a jump in differential phase TEC of more than or equal to 1.5 TECU in one second (1 TEC Unit corresponds to $10^{16}$ electrons $/ \mathrm{m}^{2}$ ).

[6] CHAIN is supported by radars, riometers, optical instruments and magnetometers of the Canadian Geospace Monitoring (CGSM) program [Liu, 2005], including the 


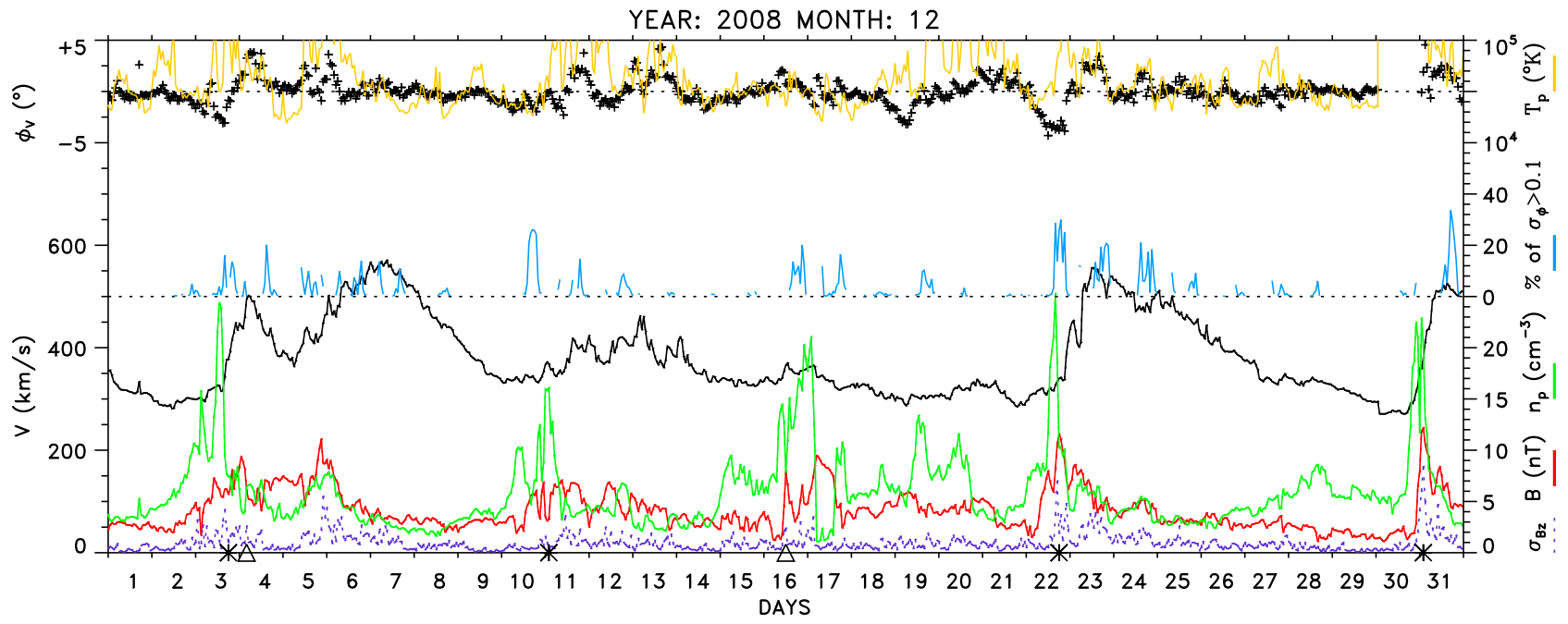

Figure 2. Hourly averages of the solar wind velocity $V$ (black line), flow angle $\phi_{V}$ (black crosses), the proton temperature $T_{p}$ (yellow), the interplanetary magnetic field magnitude $|\mathbf{B}|$ (red), solar wind density $n_{p}$ (green), and the mean standard deviation $\sigma_{B z}$ (purple dotted line) of the IMF $B_{Z}$ are shown. Asterisks and triangles at the bottom of the figure indicate CIR and ICME arrivals, respectively. Phase scintillation occurrence (blue) observed from Cambridge Bay and Taloyoak is superposed.

Northern Solar Terrestrial Array (NORSTAR) [Donovan et al., 2003].

\section{Solar Wind Data}

[7] Solar wind origins of ionospheric disturbances causing the observed scintillation are identified as either CIR/HSSs or ICMEs. For ICMEs, we use a catalog of nearEarth ICMEs [Richardson and Cane, 2010] that is updated at http://www.srl.caltech.edu/ACE/ASC/DATA/level3. Solar wind plasma parameters are obtained from the Goddard Space Flight Center Space Physics Data Facility OMNIWeb (http://omniweb.gsfc.nasa.gov/) and are used to identify arrival times of CIR/HSSs [Prikryl et al., 2009b]. In brief, as a consequence of solar rotation, a high speed stream generates a CIR, a region between the fast and slow solar wind where the solar wind plasma is compressed [Pizzo, 1978]. A stream interface is characterized by an abrupt and simultaneous drops in particle density and rises in proton temperature within [Belcher and Davis, 1971; Burlaga, 1974; Gosling et al., 1978], which is followed by a peak in magnetic field magnitude [Jian et al., 2006]. The standard deviation $\sigma_{B z}$ of the IMF $B_{Z}$ component also peaks following the stream interface and remains elevated for a few days. These signatures are associated with bipolar east-west deflections of the solar wind flow [Gosling et al., 1978]. In the geocentric solar ecliptic (GSE) coordinates, the flow angle $\phi_{V}$ increases from zero along $-\mathrm{X}_{\mathrm{GSE}}$ and is positive toward $+Y_{\mathrm{GSE}}$. The flow angle $\phi_{V}$ is often used as the solar wind parameter to determine arrival times of CIR stream interfaces [McPherron and Siscoe, 2004]. For this study, a mid-point between peaks in plasma (number) density $n_{p}$ and magnetic field magnitude $|\mathrm{B}|$ at the leading edge of rising solar wind velocity $V$ is used to identify the stream interface, the discontinuity separating slow and fast solar wind [Tsurutani et al., 2006]. This then provides the key (zero) time for superposed epoch analysis (Section 5). The flow signatures, which may differ from the latter by a few hours, were used only when peaks in $|\mathrm{B}|$ or $n_{p}$ were ill-defined or when these data were missing. A total of $64 \mathrm{HSS} / \mathrm{CIRs}$ with a maximum velocity $V_{\mathrm{MAX}}$ exceeding $500 \mathrm{~km} / \mathrm{s}$ were identified in the period from 2008 to 2010 . To characterize the solar wind by one index, we use the quasi-invariant (QI) index [Osherovich et al., 1999] that is defined as the ratio of the solar wind magnetic to ram pressures.

[8] Figure 2 shows solar wind plasma parameters in December 2008 when three major CIRs (December 3, 22 and 31) at the leading edges of HSSs $\left(V_{\mathrm{MAX}}>500 \mathrm{~km} / \mathrm{s}\right)$, and a minor CIR/HSS on December11, were observed. The CIRs at the leading edges of HSSs show peaks in $n_{p}$ and $|\mathrm{B}|$ and an increase in the mean standard deviation $\sigma_{B Z}$ of the IMF $B_{Z}$ component. The enhanced $\sigma_{B z}$ indicates enhanced amplitudes of the IMF fluctuations including solar wind Alfvén waves. Also, two ICMEs arrived on December 4 and 16 followed by decreased proton temperature (shown in yellow lines), which is one of the characteristics of ICMEs [e.g., Zurbuchen and Richardson, 2006, and references therein]. The first of these ICMEs closely followed arrival of the HSS a day before so it is not possible to distinguish its effect on scintillation from that of the CIR/HSS. The phase scintillation occurrence obtained from the Cambridge Bay and Taloyoak GPS data combined is shown (blue line) in the midsection of 

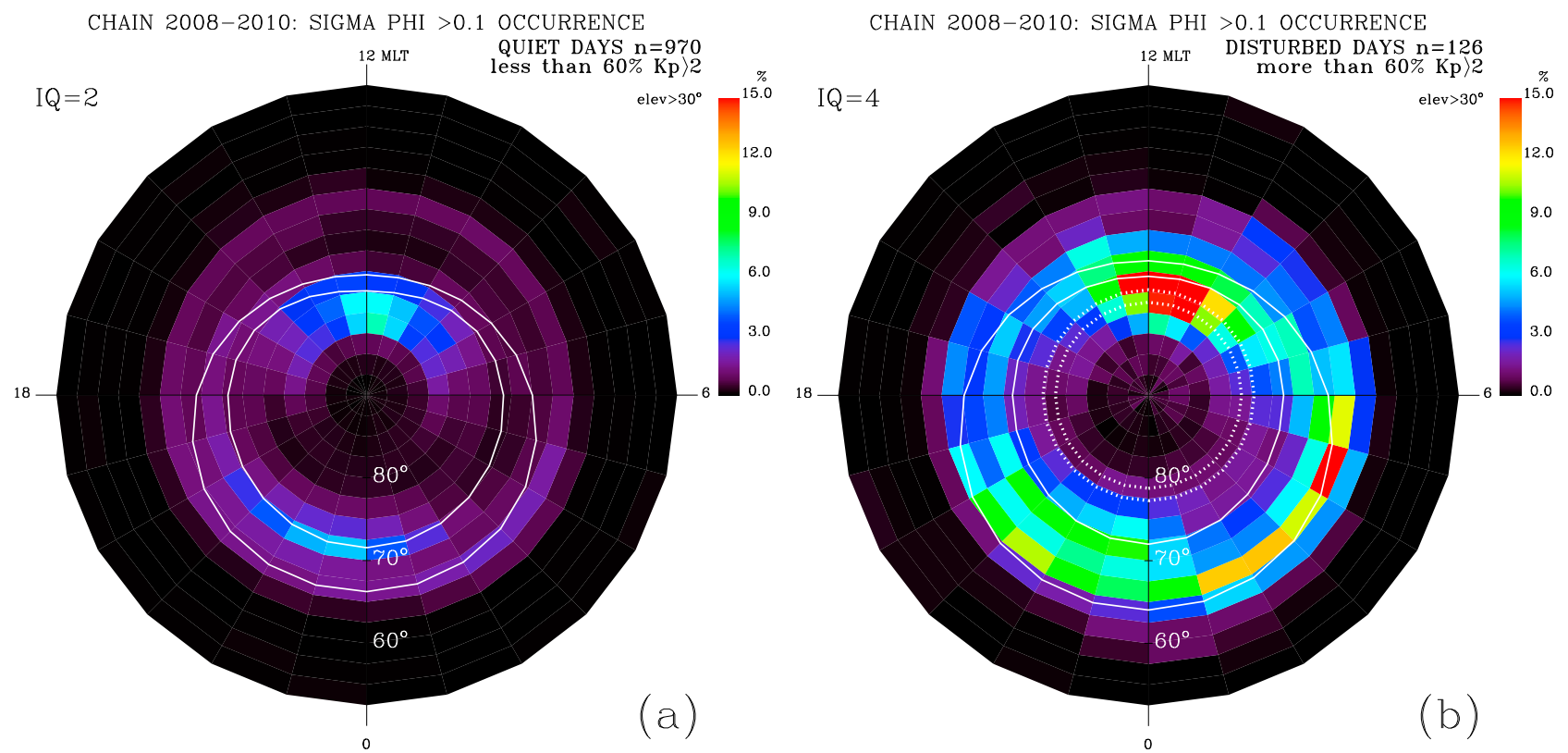

Figure 3. Phase scintillation occurrence maps for (a) low and (b) moderate Kp index.

Figure 2. The second ICME was followed by an increase in phase scintillation occurrence, as were all four CIRs shown in Figure 2.

\section{GPS Phase Scintillation at High Latitudes}

[9] At high latitudes, the maximum occurrence of GPS phase scintillation was found in the auroral oval near magnetic midnight [Aarons, 1997] particularly during magnetic storms [Aarons et al., 2000]. However, phase scintillation is known to also occur on the dayside in the cusp and in the polar cap [Basu et al., 1998; Spogli et al., 2009; Alfonsi et al., 2011; Prikryl et al., 2011a, 2011c]. A climatology study for solar minimum years of 2008 and 2009 [Prikryl et al., 2011a] showed that phase scintillation, as a function of magnetic local time and geomagnetic latitude, primarily occurs on the dayside in the ionospheric footprint of the cusp and on the night-side in the auroral oval. As expected, these scintillation regions shift in latitude in response to varying geomagnetic activity. The statistics have been extended to 2010 [Prikryl et al., 2011b] when the solar activity started to increase in the new solar cycle. Figures $3 a$ and $3 b$ show the maps of the percentage occurrence of phase scintillation with $\sigma_{\Phi}$ exceeding 0.1 radians as a function of magnetic local time (MLT) and CGM latitude for 970 quiet and 126 disturbed days selected using $K p=2$ as a threshold. Superposed on the maps are the boundaries of the Feldstein statistical auroral oval for quiet and moderately disturbed conditions [Holzworth and Meng, 1975]. As expected, the scintillation occurrence is confined in a smaller and narrower area during quiet conditions. The scintillation area becomes larger and broader when the auroral oval expands during disturbed conditions. It is noted that the phase scintillation occurrence map in
Figure $3 b$ is qualitatively similar to the observed and modeled height-integrated electron-density irregularity strength from the WideBand Model (http://www.nwra. com/ionoscint/wbmod.html) for moderate solar activity level (SNN 50 to 75), which is much higher than the average SNN during the last deep solar minimum. The similarity in the latitudinal and MLT extent of enhanced scintillation in the cusp and auroral oval is also found when comparing with the modeling results by Wernik et al. [2007, Figure 13].

[10] Dotted concentric circles in Figure 3b show latitudes of Cambridge Bay (CBB) and Taloyoak (TAL). The CBB data were available since December 2007 while TAL started operation in July 2008. The combined CBB + TAL data set samples the poleward edge of auroral oval in the night [Prikryl et al., 2010] but is representative of the cusp ionosphere during the daytime. The combined CBB + TAL time series of phase scintillation occurrence are used to examine correlation with solar wind parameters and to examine the possibility of forecasting phase scintillation following CIR/HSSs and ICMEs.

[11] We now compare the scintillation occurrence over the CHAIN array during days of active ICMEs (Figure 4a) and HSS/CIRs (Figure $4 \mathrm{~b}$ ) with the geomagnetically disturbed days based on $K p$ index as shown in Figure $3 \mathrm{~b}$. The catalog of near-Earth ICMEs [Richardson and Cane, 2010] includes 27 ICME events from 2008 to 2010 (50 days in total). It is noted that only a few of these ICMEs caused significant geomagnetic storms (minimum Dst of -123 nT) and intense scintillation events [e.g., Prikryl et al., 2011c]. As shown in Figure 4a, the phase scintillation during these 50 ICME days was observed mainly in the cusp and in the auroral oval. As the auroral oval expanded during disturbed conditions after the arrival of ICMEs the area of 

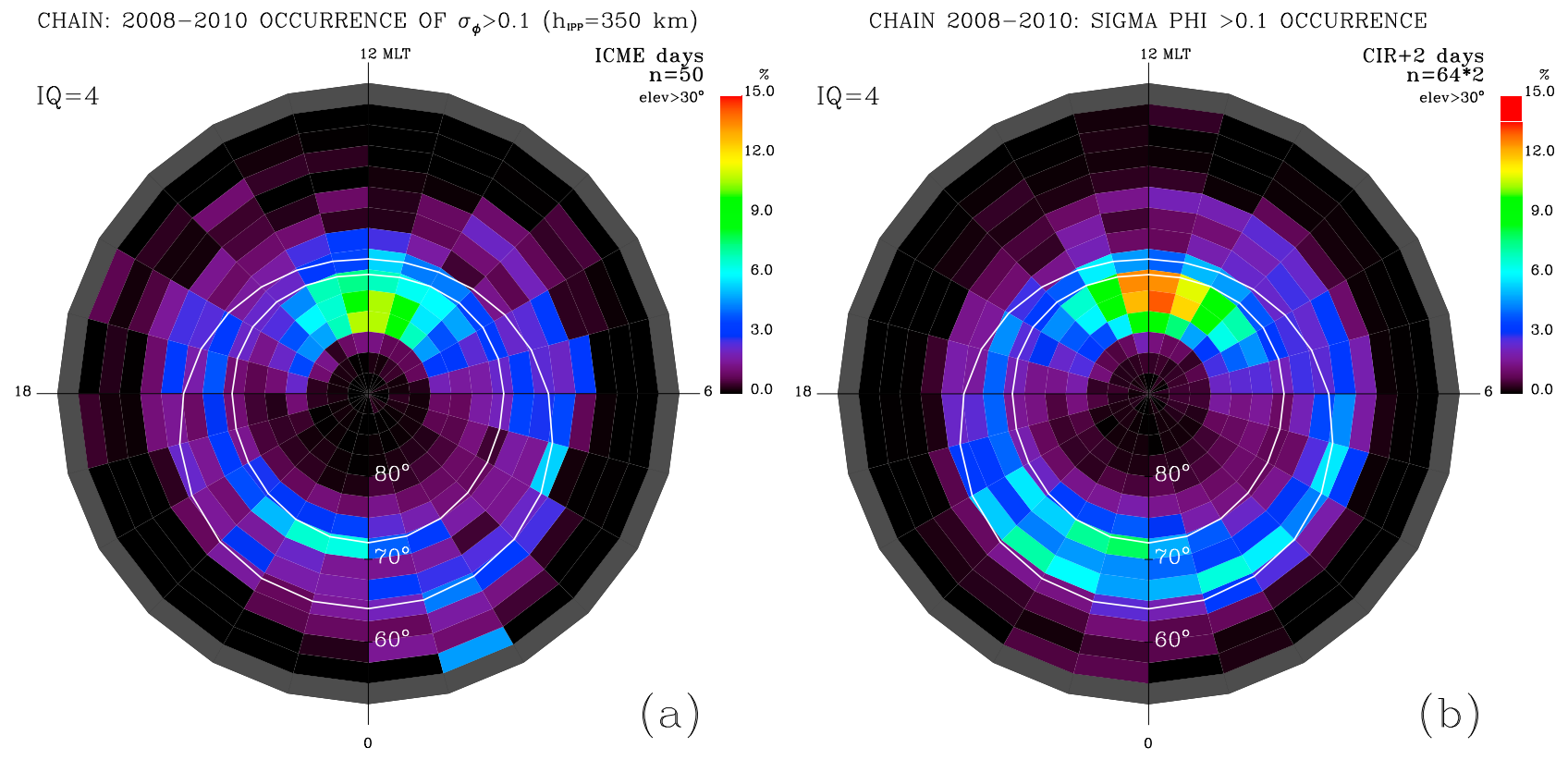

Figure 4. Phase scintillation occurrence maps for (a) ICMEs and (b) CIRs/HSSs.

scintillation occurrence become broader. Also, the percentage occurrence (Figure 4a) was significantly greater than for all quiet days (Figure 3a), particularly in the cusp, but it was about half of the occurrence for all geomagnetically disturbed days (Figure $3 b$ ). For 128 CIR/HSSs days (two days at the start of each 64 HSSs, i.e., the CIR day and the following day), the phase scintillation occurrence (Figure $4 \mathrm{~b}$ ) was higher than for ICME days (Figure 4a) but lower than for all geomagnetically disturbed days (Figure 3b), both in the cusp and in auroral oval. Clearly, the geomagnetically disturbed days included other scintillation active days than the major CIR/HSS days. These include the strongest ICMEs, other disturbed days during major HSSs after the CIR + 2 day, and some minor CIR/ HSS $\left(V_{\mathrm{MAX}}<500 \mathrm{~km} / \mathrm{s}\right)$ like the one on December 10, 2008 (Figure 2).

\section{The Superposed-Epoch Analysis of Solar Wind Plasma Parameters}

[12] As shown in Figure 2, arrivals of high-speed streams (HSS) originating from coronal holes are followed by significantly enhanced phase scintillation occurrence particularly in the cusp, usually continuing for a few days. To obtain the mean response of scintillation relative to CIR arrival time we applied superposed epoch (SPE) analysis on time series of scintillation occurrence and solar wind parameters using key times of 64 CIRs. Figure 5a shows typical mean variations of the solar wind parameters over 12 days centered at the key time (day 0 ). The mean solar wind velocity decreases to a minimum just before the key time and then steeply rises to a maximum two days later. All other mean solar wind parameters peak at, or very close to, the key time. The mean flow angle $\phi_{V}$ shows a bipolar east-west deflection. Figure $5 \mathrm{~b}$ shows that the mean phase scintillation occurrence steeply rises at the CIR arrival day tapering off a few days later, which is similar to the mean QI and Kp indices. Also, the average number of cycle slips, which is nearly zero a few days before the CIR key day increases at the CIR interface and remains elevated during the HSS. A strong 1-day periodicity in scintillation CBB + TAL time series is due to cusp location of these stations where the scintillation maximizes and is only partly smoothed out in this relatively small statistical sample of $64 \mathrm{CIRs}$.

[13] Figures $6 a$ and $6 b$ show the results of the superposed epoch (SPE) analysis for 27 ICMEs. Because the ICMEs are often preceded by shocks, the mean $V, n_{p},|\mathbf{B}|$ and $\sigma_{B z}$ increase near or just before the key time although the peak values are much lower than for CIRs. The QI index increases more gradually and to higher values than for CIRs. This is because many ICMEs are actually magnetic clouds and the ratio of the magnetic to ram pressures is higher inside the magnetic cloud. The $K p$ index peaks at a mean value of only 2. Also, the mean response of cycle slip number and phase scintillation occurrence relative to ICME arrivals is weaker than for CIRs for the full data set of ICMEs. However, if weak ICMEs are eliminated by selecting more geo-effective ICMEs (Dst $<-10 \mathrm{nT}$ ), the increase in the mean phase scintillation occurrence is about 1.5 times larger (Figure 6c) than when no selection is made, and comparable to the increase after major CIR/ HSSs (Figure 5b). The mean number of cycle slips also increases at the ICME arrival although the reduced number of epochs averaged is too small to suppress other unrelated random events. If the SPE analysis is further 

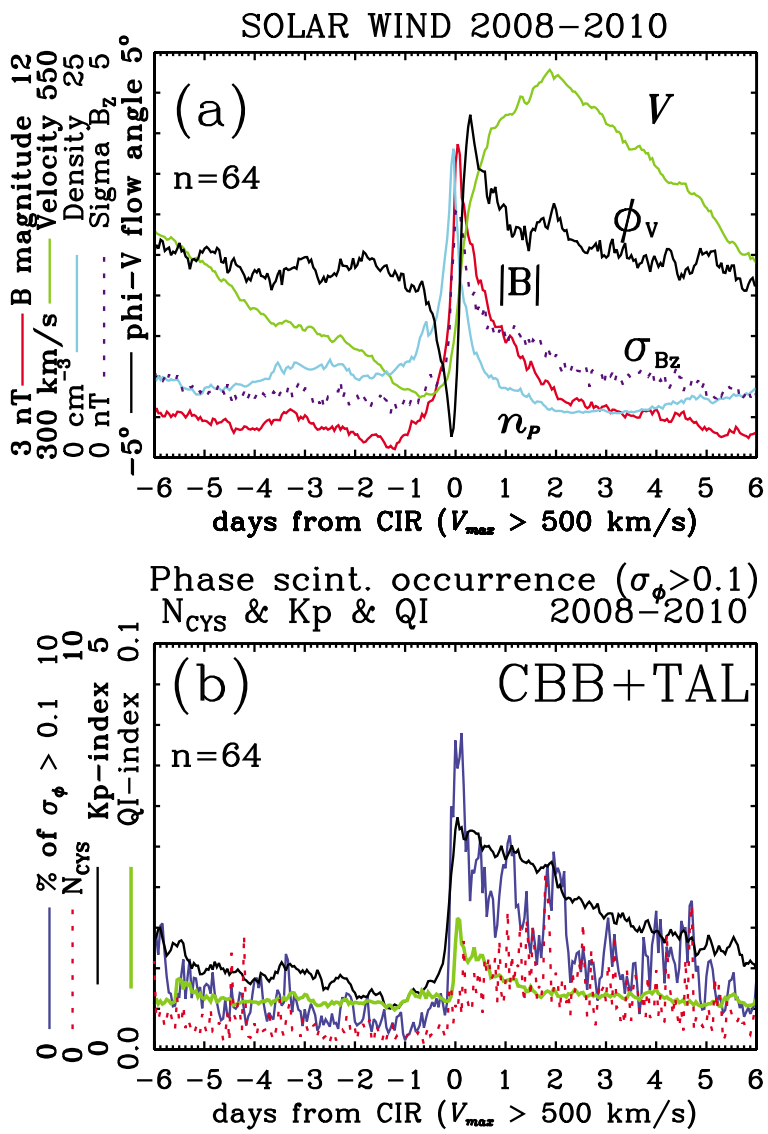

Figure 5. Superposed epoch analysis of (a) solar wind plasma parameters $V, n_{p},|\mathbf{B}|, \sigma_{B z}$ and $\phi_{V}$ and (b) QI, $\mathrm{Kp}$, number of cycle slips $N_{c y s}$ and phase scintillation occurrence from the Cambridge Bay and Taloyoak scintillation data combined. All time series are keyed by the times of major CIRs.

limited to only 7 strongest ICMEs (Dst $<-30 \mathrm{nT}$ ) (not shown) the peak mean scintillation occurrence and $K p$ index are $\sim 12 \%$ and 3.5, respectively.

\section{Recurrent HSSs and Daily Occurrence of Phase Scintillation in the Cusp}

[14] Systematic behavior of recurrent HSSs that originate from coronal holes is well known and has become the basis of forecasting methods of space weather including geomagnetic activity [McPherron and Siscoe, 2004; McPherron and Weygand, 2006]. The time separation of successive streams can often be determined by the heliospheric longitude separation of equatorial coronal holes (heliographic latitude $<40^{\circ}$ ), which is commonly $360^{\circ} / n$, where $n=1,2,3$ or 4 . There may be 1 to 4 recurrent coronal holes per solar rotation ( $\sim 27.3$ days as viewed from the Earth) and thus typical time separations of major recurrent plasma streams are $\sim 27,13.5,9$ and 7 days. As a result, such periodicities are often found in the solar corona, solar wind as well as in
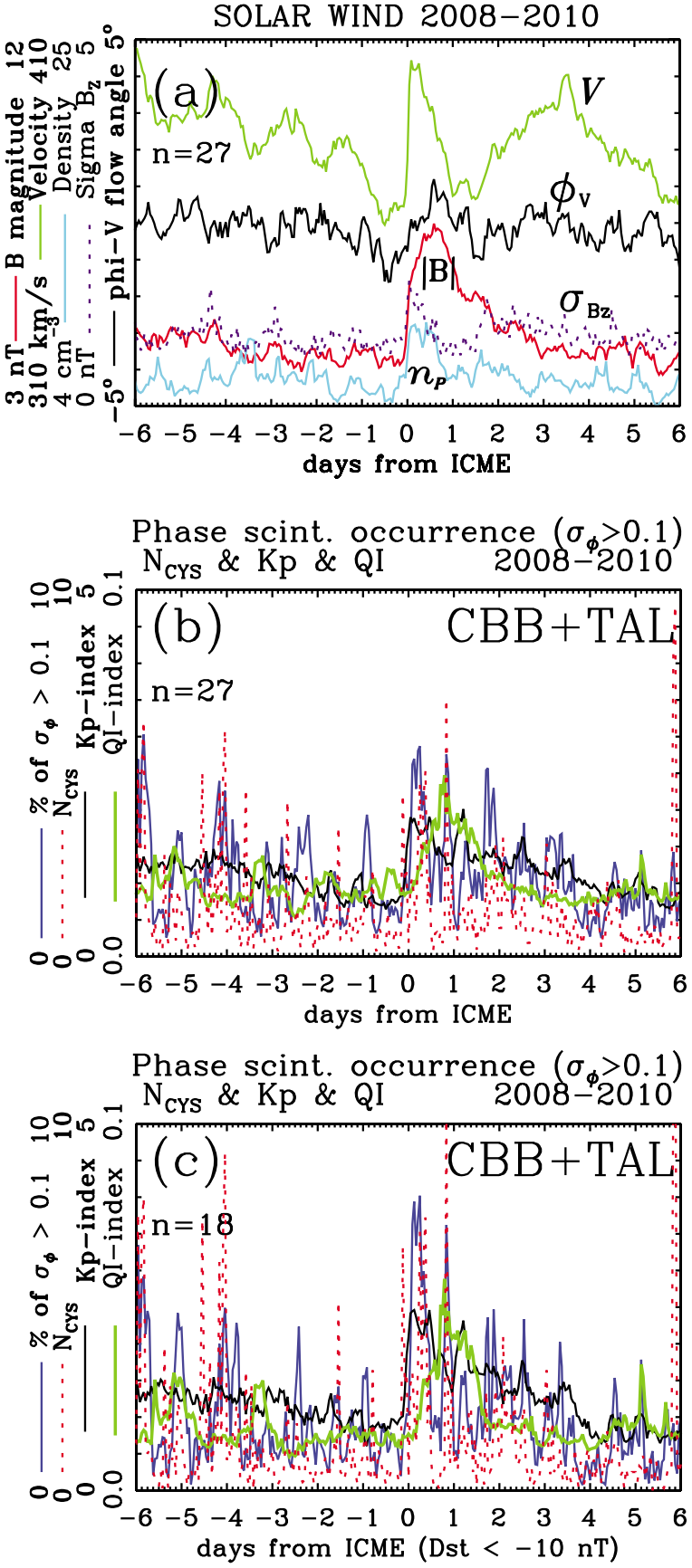

Figure 6. Superposed epoch analysis of (a) solar wind plasma parameters $V, n_{p},|\mathbf{B}|, \sigma_{B z}$ and $\phi_{V}$ and (b and c) QI, Kp, number of cycle slips $N_{c y s}$ and phase scintillation occurrence from the Cambridge Bay and Taloyoak scintillation data combined. The time series are keyed by the start times of ICMEs. 

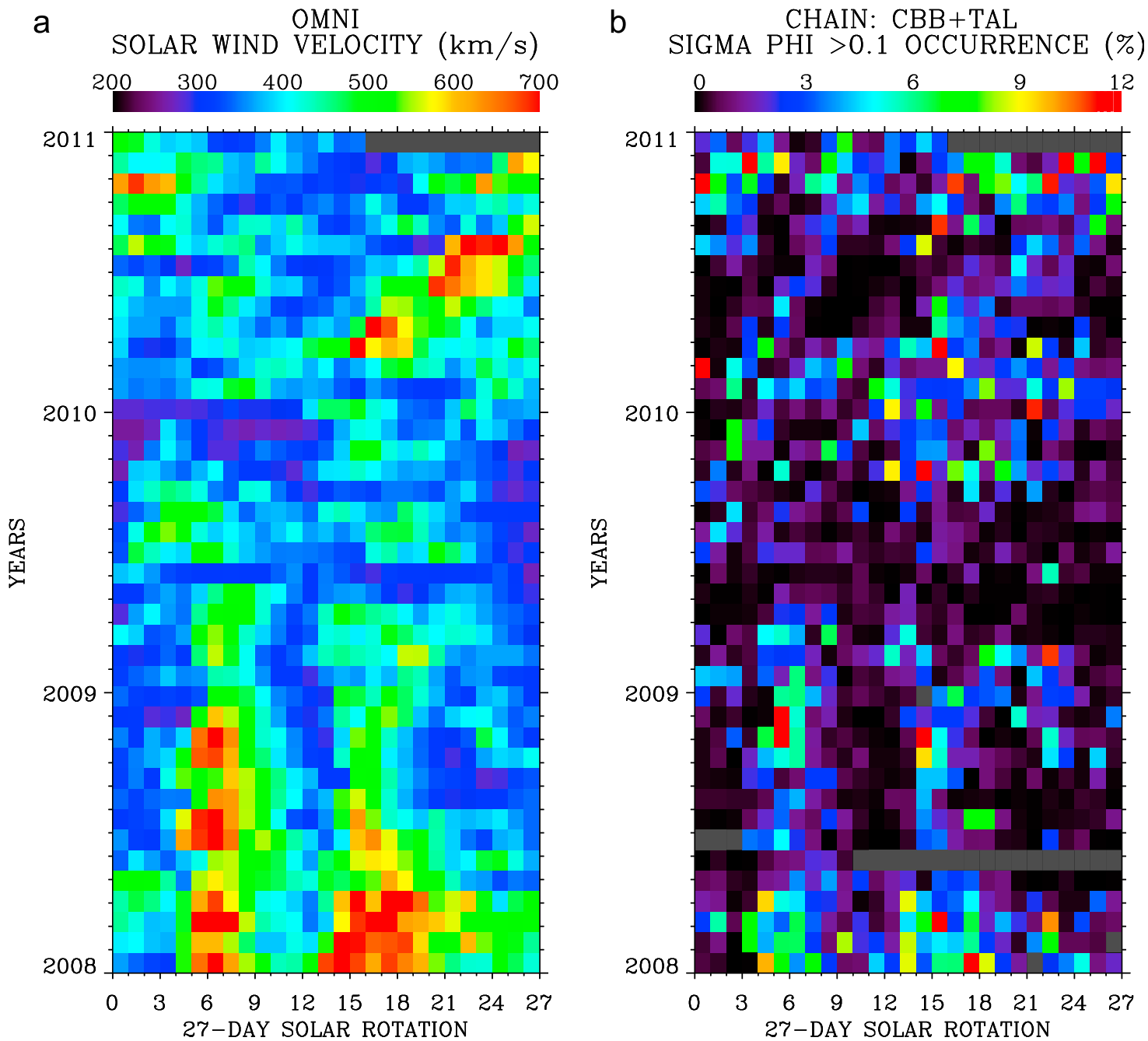

Figure 7. Daily averages of (a) solar wind velocity and (b) phase scintillation occurrence in Cambridge Bay and Taloyoak.

the geophysical data including geomagnetic, ionospheric and sometimes even atmospheric indices and parameters [e.g., Mlynczak et al., 2008; Thayer et al., 2008; Prikryl et al., 2009a; Sojka et al., 2010; Emery et al., 2011; Verkhoglyadova et al., 2011; Love et al., 2012]. However, differential solar rotation is well known for the photosphere, and can also manifest itself in the corona [see, e.g., Shnirman et al., 2010, and references therein]. While the differential rotation of coronal holes has been confirmed in general, a different behavior was found between the isolated equatorial coronal holes and the extensions of polar holes. A rotation rate in the corona does increase toward the equator below $40^{\circ}$ of heliographic latitude and can be more rigid there than in the photosphere but it increases toward the poles above the same heliographic latitude [Navarro-Peralta and Sanchez-Ibarra, 1994; Insley et al., 1995].

[15] Recurrent HSSs and the response of the ionosphere can be best viewed in a stack plot of time series every 27 days. Figures $7 \mathrm{a}$ and $7 \mathrm{~b}$ show daily averages of solar wind velocity and phase scintillation occurrence for years 2008 to 2010 starting on January 1, 2008. Two recurrent HSSs that persisted for more than a year and a half are seen in Figure 7a around days 6 and 16. In 2009, they were diminished in velocity as the equatorial coronal holes started to shrink, while midlatitude coronal holes formed [Tsurutani et al., 2011, and references therein]. In 2010, this regular structure has changed as the activity of the new solar cycle 24 including CMEs has begun to appear. However, recurrent streams from slower rotating coronal holes can be seen as tilted bands in the solar wind velocity plot. In 2010, there were some isolated coronal holes at high-latitude, as well as polar coronal holes that extended to lower heliospheric latitudes that were joined at heliographic equator. In contrast, the HSSs in 2008 originated mostly from large recurrent equatorial coronal holes resulting in high peak velocities and large amplitude Alfvén waves [Tsurutani et al., 2011]. 

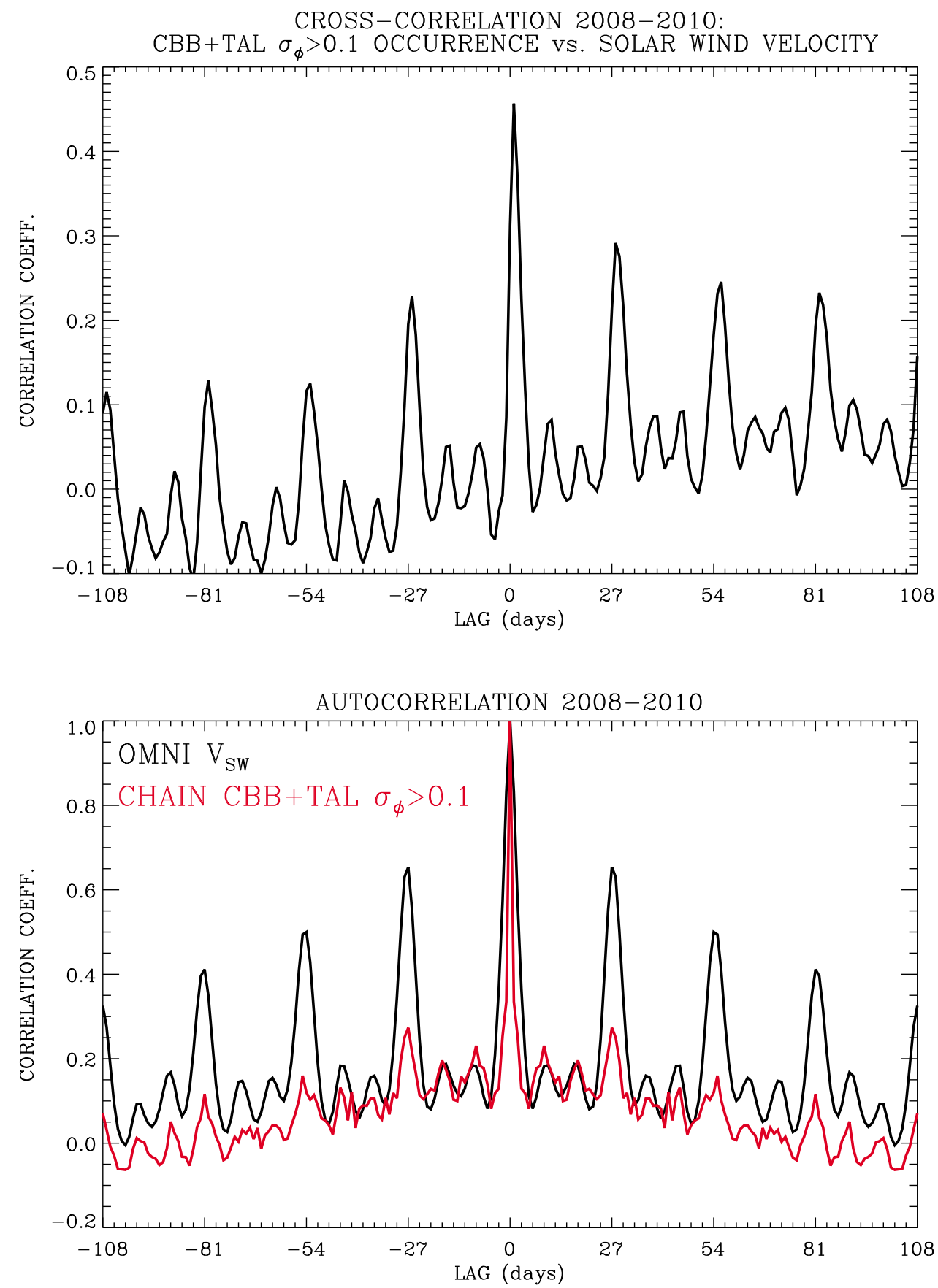

Figure 8. Cross- and auto-correlation of daily averages of phase scintillation occurrence and solar wind velocity.

[16] In response to solar wind HSSs the enhanced phase scintillation at CBB and TAL showed a very similar temporal pattern (Figure 7b). From January 2008 to May 2009, the scintillation occurrence was closely associated with two major recurrent HSSs although other minor HSSs persisted for several rotations. In 2010, scintillation was again associated with HSSs but this time the HSSs co-rotated with high-latitude coronal holes at about 28-day rotation period.

[17] The overall (2008-2010) cross-correlation between the average daily solar wind velocity and the scintillation occurrence (Figure 8a) not only shows a peak of 0.46 at lag 0 but also peaks at multiples of solar rotation. Likewise, the autocorrelation functions (Figure 8b) for both time series show the presence of solar periodicity of 

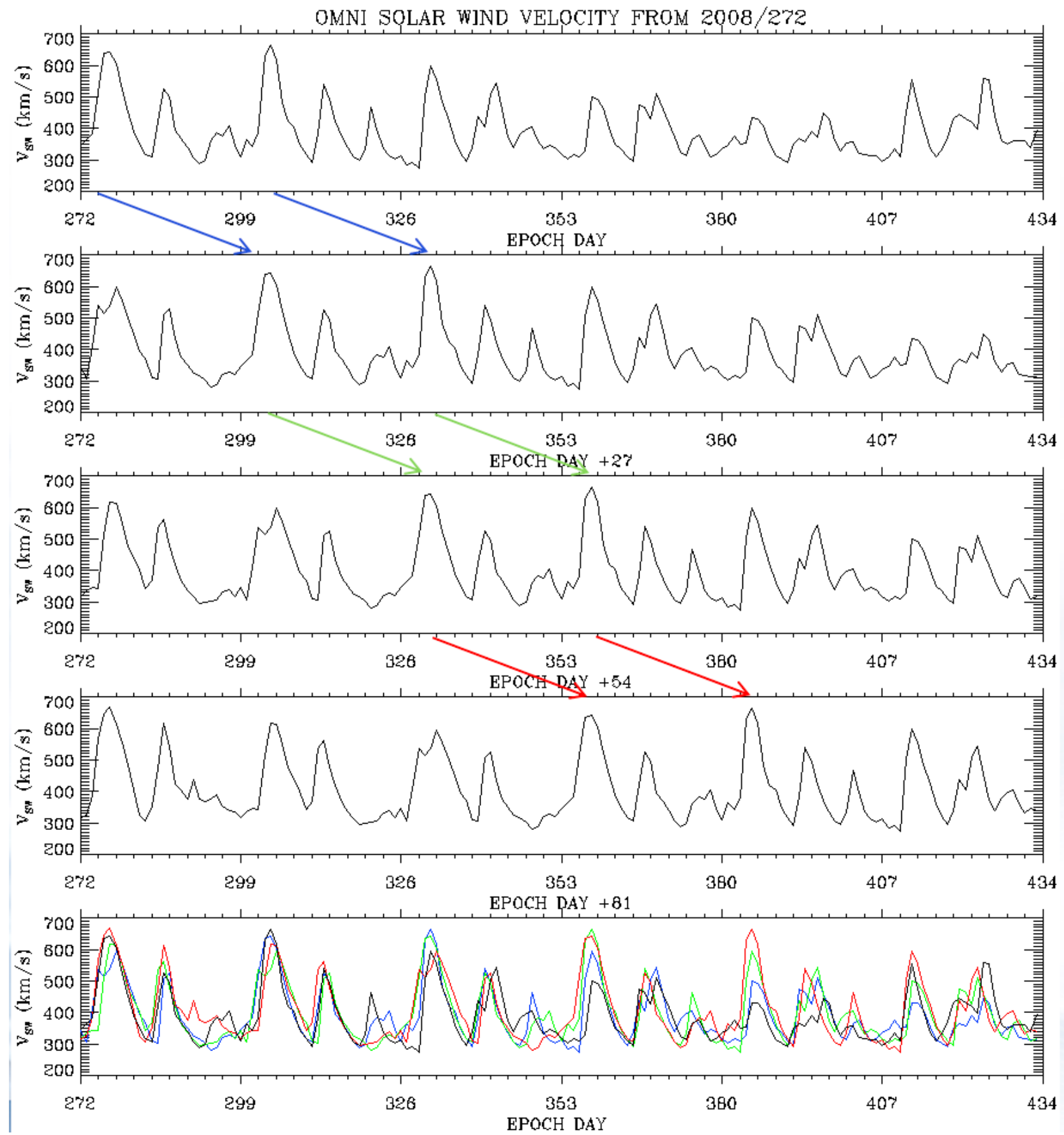

Figure 9a. The daily solar wind velocity over 6 solar rotations from September 28, 2008 to March 9, 2009 (first panel). The second, third, and fourth panels show "forecasts" by shifting the time series in time by 27,54 , and 81 days. The fifth panel is a superimposition of all the above panels.

approximately 27 days. These peaks and two subsidiary peaks, i.e., a triple peak per solar rotation, are consistent with occasional presence, on average, of 3 active HSSs (coronal holes) per solar rotation (Figure 9a). The 9-day recurrence of geomagnetic activity correlated with solar wind velocity was most prominent in 2008 , with the 7 -day (more precisely 6.7-day) periodicity present in the preceding years, particularly in 2006 [Love et al., 2012]. 

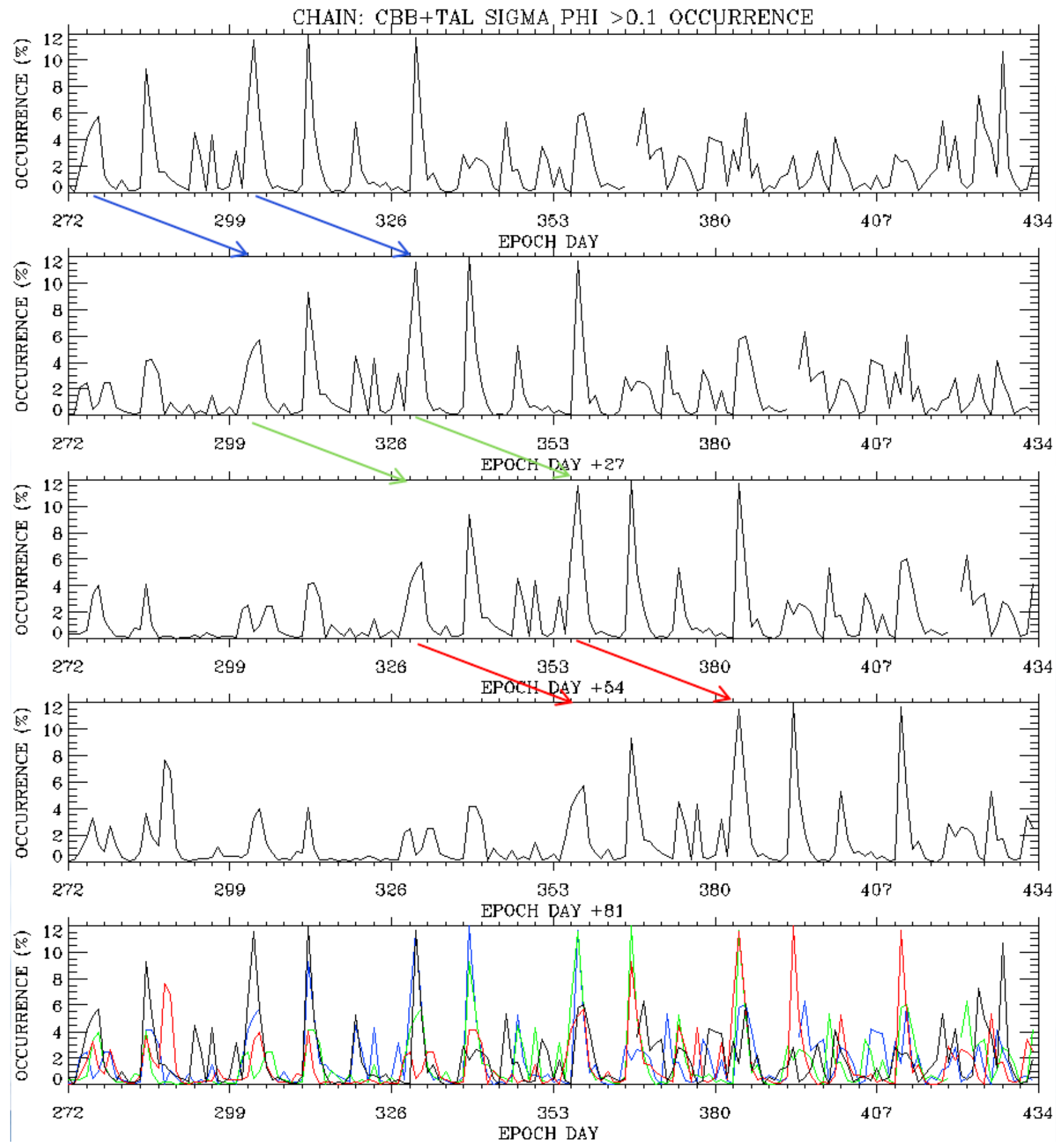

Figure $9 \mathrm{~b}$. The daily occurrence of phase scintillation over 6 solar rotations from September 28, 2008 to March 9, 2009 (first panel). The second, third, and fourth panels show "forecasts" by shifting the time series in time by 27,54 , and 81 days. The fifth panel is a superimposition of all the above panels.

[18] The feasibility of forecasting the solar wind conditions and scintillation occurrence for one to multiple solar rotations ahead during a period of stable recurrent HSSs streams is illustrated in Figures $9 a$ and $9 b$. During this period two major and a minor HSSs were observed. By shifting the time series successively by 27 days the past data can be used as "forecasts" for multiple solar rotations ahead. Thus "forecasted" major and even some minor 

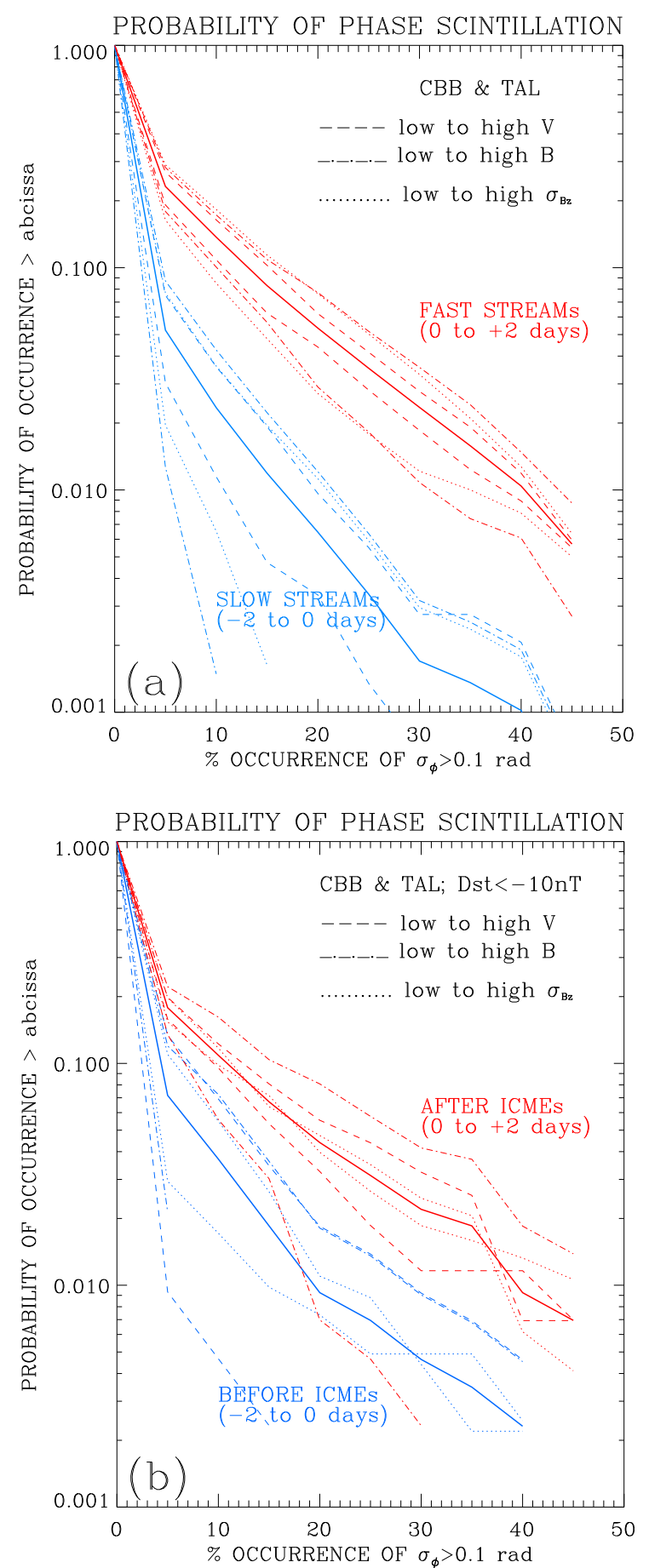

Figure 10. The cumulative probability distribution functions for the phase scintillation occurrence combined for TAL and CBB during (a) slow and fast solar wind up to two days before and after CIRs $(\mathrm{n}=64)$, and (b) two days before and after ICMEs (Dst < $10 \mathrm{nT}$; $n=18$ ). The overall probabilities for each case (solid lines) are bracketed by a pair of curves (broken lines) for data corresponding to low and high (below and above median) solar wind parameter values. peaks in solar wind velocity and phase scintillation occurrence show reasonably good agreement with the observed (unshifted) time series up to 3 solar rotations ahead.

\section{Probabilistic Forecasting of Phase Scintillation in the Cusp Based on the SPE Analysis of Solar Wind CIRs and ICMEs}

[19] McPherron and Siscoe [2004] and McPherron and Weygand [2006] demonstrated a new promising technique to forecast geomagnetic disturbance indices relative to the passage of the stream interface between fast and slow solar wind. In particular, they showed the geoeffectiveness of what they called "corotating compression ridges," their reference to CIRs. McPherron and Siscoe [2004] construed their method of probabilistic forecasting of geomagnetic activity due to specific solar wind conditions as an analogy to traditional meteorology approach in weather forecasting based on atmospheric air mass climatologies. In this analogy, a stream interface between fast and slow solar wind (a CIR or an ICME magnetosheath), which leads to geomagnetic storms, corresponds to a boundary (weather front) between atmospheric air masses where the weather storms develop. By applying the "air mass" concept to space weather forecasting McPherron and Siscoe [2004] provided proof of concept of the method and discussed steps to improve it.

[20] This method is readily applicable to other manifestations of space weather in the geospace environment that are sensitive to arrivals of HSSs, including ionospheric scintillation. Figure 10a shows the cumulative probability distribution function (PDF) for the phase scintillation occurrence at TAL and CBB contrasting the periods of calm [Borovsky and Steinberg, 2006] during two days of slow solar wind just before the major CIR/HSSs arrivals, and two "stormy" days of fast solar wind disturbances that followed. The probability that phase scintillation will exceed a given value as plotted on the abscissa is shown. The overall probability curves for both the slow and fast streams (shown by solid lines) are bracketed by probability curves (broken lines) for scintillation data subdivided by corresponding solar wind low and high (below and above median) values of $V,|\mathbf{B}|$ and $\sigma_{B z}$. Likewise, Figure $10 \mathrm{~b}$ shows the probabilistic curves for the SPE analysis of 18 ICMEs (Dst $<-10 \mathrm{nT}$ ) for two days before and after ICME arrivals.

[21] As can be seen in Figure 10a, probability of scintillation occurrence is low before but significantly increased immediately after the CIR arrival. The probability of scintillation occurrence also depends on solar wind plasma parameter values. The PDFs results are very similar for days after ICMEs (Figure 10b), although the differences between the probabilities before and after ICME arrival are smaller. Unlike CIRs, ICMEs are not necessarily preceded by a particularly quiescent period. For example, there may be ongoing activity associated with a preceding ICME, and enhanced activity may arise from the interaction of an 

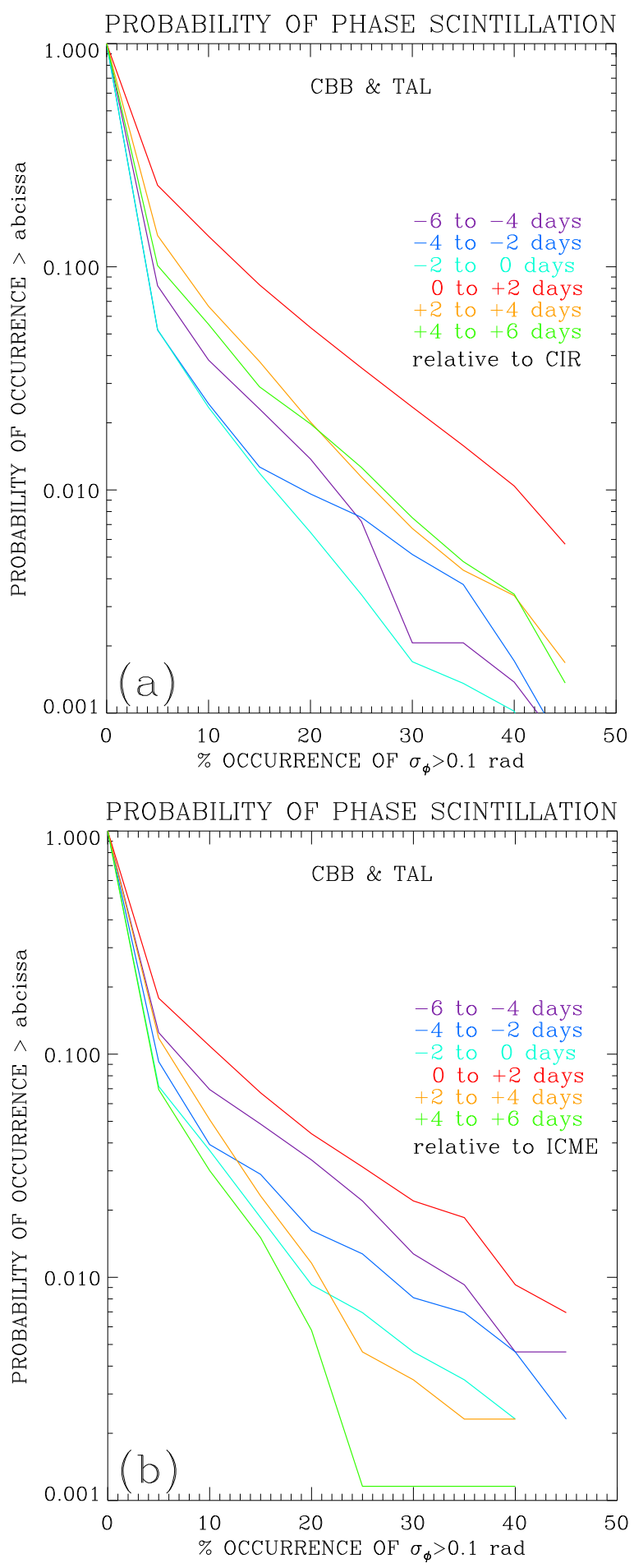

Figure 11. The cumulative probability distribution functions for the phase scintillation occurrence combined for TAL and CBB stepped by 2-day intervals before and after (a) CIRs $(n=64)$ and (b) ICMEs (Dst < $10 \mathrm{nT} ; \mathrm{n}=18$ ).
ICME with the background solar wind. Figures 11a and $11 \mathrm{~b}$ show PDFs stepped by 2-days intervals before and after CIRs and ICMEs. The probabilities are clearly highest for two days after CIR and ICME arrivals. Daily PDFs can be generated but for clarity only 2-day PDFs are shown.

[22] Because the effects of HSS/CIRs can last for several days it is expected that the scintillation would also persist longer than after the arrival of ICMEs. This and the contrast between the scintillation level during "calm" and "stormy" days before and after CIR arrivals is demonstrated in Figures $5 \mathrm{~b}$ and $11 \mathrm{a}$. The ICME effects can be very intense but are usually short-lived, with the intensity of the ICME impact and its duration depending on the IMF direction and the ICME speed. Because of a very small number of epochs superposed, Figures $6 \mathrm{~b}$ and $6 \mathrm{c}$ are too noisy to show that. As a result, the PDFs shown in Figure 11b do not reliably represent the probabilities for "quiet" days before ICME, the mean peak probabilities on the day of ICME arrival, or the actual duration of the ICME effect. Larger data sample of moderate to strong ICMEs is needed to provide that and to suppress the contamination by HSS/ CIRs and other disturbances.

\section{Discussion}

[23] In this paper, we applied the forecasting method by McPherron and Siscoe [2004] to forecasting phase scintillation occurrence in the high-latitude ionosphere typical of a magnetospheric cusp footprint during a solar minimum. These results demonstrate the feasibility of probabilistic forecasting of scintillation; however, more extensive databases with improved spatial coverage in latitude/longitude to include polar cap and auroral oval, as well as coverage of a complete solar cycle, are needed. In particular, the majority of ICMEs during the last solar minimum caused only weak geomagnetic disturbances and none resulted in a major geomagnetic storm. Thus this sample of ICMEs is clearly not representative of periods of higher solar activity. In comparison, strong and recurrent high-speed streams from coronal holes remained active during most of the period studied in this paper. The obtained mean probability curves for days before and after CIR arrival are expected to be similar throughout the solar cycle, although they may reflect the differences between slow and fast solar wind that are likely to be enhanced during the declining phase when the recurrent streams are dominant.

[24] When a larger statistical sample becomes available the concept of probabilistic forecasting of scintillation will be further refined by compiling probability curves separately for different seasons, levels of solar activity, various threshold values of $\sigma_{\Phi}$, as well as different magnetic latitudes and local times. It will be important to distinguish between the periods of southward and northward IMF. The IMF $B_{Z}$ component (in the geocentric solar magnetospheric coordinate system, GSM) controls the coupling to the magnetosphere and the energy deposition into the ionosphere. Considering the Russell-McPherron effect [Russell and McPherron, 1973], CIRs can be classified by 

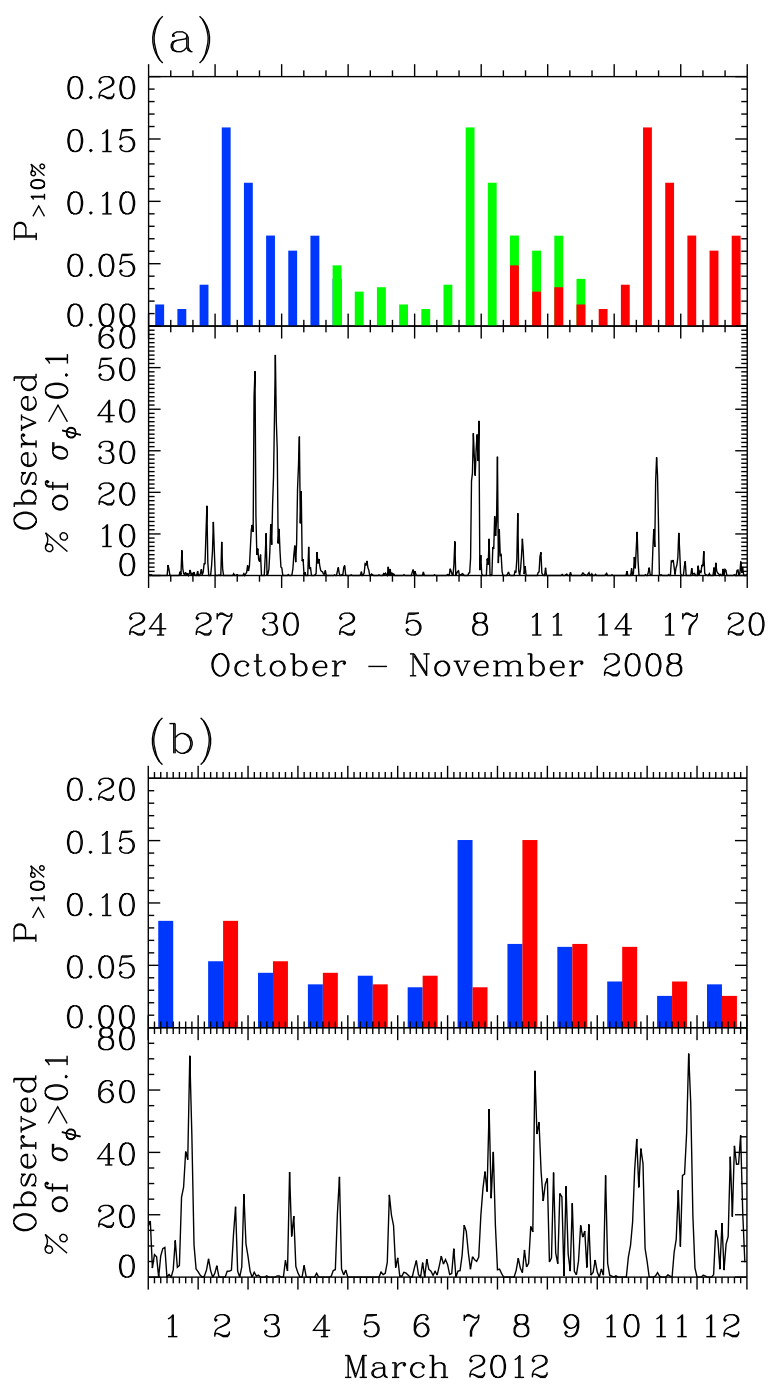

Figure 12. Examples of probabilistic forecasting of phase scintillation occurrence following the anticipated arrivals of (a) HSSs/CIRs and (b) ICMEs. The values of the cumulative probability distribution functions for \pm 6 days relative to the solar wind disturbance arrival day are used to obtain daily probabilities of phase scintillation occurrence to exceed $10 \%$. The observed hourly occurrence of phase scintillation is shown in the bottom plots.

IMF polarity as shown by Miyoshi and Kataoka [2008] and adopted by Prikryl et al. [2009a, 2009b]. For months around the spring and fall equinoxes, the mean IMF $B_{Z}$ component systematically shifts southward near the CIR interface for the group of events classified as type SBZ (southward $B_{Z}$ ), while the CIRs are associated with a northward shift in $B_{Z}$ for the group classified as NBZ (northward $B_{Z}$ ). If adopted for probabilistic forecasting of scintillation, this classification of CIRs should result in higher and lower probabilities for SBZ and NBZ groups, respectively.
[25] Although in this paper we focus on high latitudes, it is appropriate to comment on the HSS effects at the low latitudes. It has been shown that TEC at low and midlatitudes is affected by HSSs [e.g., Verkhoglyadova et al., 2011]. During disturbed periods the Joule heating at high latitudes controls neutral wind circulation that impacts the ionosphere at all latitudes and affects storms development. Also, there is direct penetration of the electric field to low latitudes as well as equatorward propagating traveling ionospheric disturbances (TIDs), both of which can result in enhanced ionospheric irregularity production at low latitudes. Although the occurrence of post-sunset scintillation at low latitudes is known to be more unpredictable than scintillation at high latitudes, the proposed method may have some value in forecasting of scintillation at low latitudes. This could be investigated by applying the SPE analysis on low-latitude scintillation time series.

[26] Probabilistic forecasting of scintillation relative to solar wind disturbances ultimately requires accurate prediction of CIR or CME arrivals. Space weather forecasting has benefited from recent advances in MHD modeling of CMEs and CIRs [Odstrcil et al., 2005; Wu et al., 2011] as well as multispacecraft tracking of CIRs propagation [Williams et al., 2011; Davis et al., 2012]. Combining the solar wind modeling and monitoring efforts with improved space weather forecasting will lead to more realistic forecast of ionospheric response including scintillation occurrence and other stochastic phenomena modulated by solar wind.

[27] To illustrate a possible application of PDFs shown in Figures 10a and 11a in forecasting scintillation occurrence we choose three CIRs identified in hourly OMNIWeb data in 2008 on September 30 ( $\sim 17$ UT), October 11 ( $\sim 8$ UT) and October 19 (22 UT) from the interval shown in Figure 9. As already discussed in Section 6, such stable recurrent HSSs streams can be forecast at least for one solar rotation, particularly in the absence of ICMEs. Applying the method of probabilistic forecasting one can propagate daily probabilities of scintillation occurrence to exceed, for example, $10 \%$ for \pm 6 days relative to October 27 , November 7 and 15 (0 UT). This would provide a scintillation occurrence forecast for a 27 day period composed of forecasts for individual CIRs (Figure 12a). For days with multiple PDF values due to overlapping 12-day intervals the maxima may be taken as a forecast for a given day. Individual forecasts could be updated and fine-tuned at a later date as more data become available, as coronal holes reappear on the east solar limb, solar wind models are updated and eventually the CIRs are observed upstream of the solar wind (e.g., ACE). The observed hourly scintillation occurrence is shown in Figure 12a (bottom). The corresponding daily scintillation occurrence values that are shown in Figure 9b (first panel; days 298-325) are consistent with daily probabilities expressed in percentages. Clearly, the simple approach using 27-day recurrence period does not produce quite satisfactory result for the first CIR, in which case the scintillation is predicted one day too early. This could be improved if more precise time 
of CIR is considered but variability in the rotation period of coronal holes is expected, as discussed in Section 6.

[28] In principle, similar forecasts of scintillation probabilities for ICMEs could be attempted and added to CIR forecasts, of course, with shorter lead times. As an example, we consider recent events of two CMEs triggered by X-class solar flares in 2012 on March 5 at 03:30 UT and on March 7 at 00:02 UT. The solar wind including the ICMEs was modeled at the Goddard Space Weather Lab http:// iswa.gsfc.nasa.gov/iswa/iSWA.html. The model predicted the impact of the ICMEs at the Earth for early UT hours on March 7 and 8, respectively (http://earthsky.org/space/ another-major-solar-flare-during-night-of-march-6-7-2012). Using the CELIAS/MTOF proton monitor on the SOHO spacecraft (http://umtof.umd.edu/pm/), the approximate travel times of the first and second ICME were $\sim 49$ and $35 \mathrm{~h}$, respectively. Based on solar wind data projected to the bow shock (1-min OMNIWeb data), the magnetospheric impacts occurred on March 7, 04:30 UT and March 8, 11:00 UT. However, in this exercise of nowcasting we adopt the solar wind model predictions of the ICME impact times on March 7 and 8, consult the statistical probabilistic curves (Figure 11b) to obtain daily probabilities of interest and use them to "predict" daily occurrence of phase scintillation in the cusp. Figure $12 \mathrm{~b}$ shows two identical sets of daily probabilities of scintillation occurrence to exceed $10 \%$ for \pm 6 days centered on March 7 and 8 superposed in blue and red colors, respectively. The observed hourly scintillation occurrence at Cambridge Bay is shown in Figure 12b (bottom) (no data were available from Taloyoak). It should be noted that the probabilities in this example are based on a very small sample of ICMEs (Figure 11b), in which very few ICMEs matched the magnitude of the March 2012 ICMEs. More realistic forecasts will require extensive database covering at least one solar cycle, which may provide a possibility to discriminate among the intensity levels of CMEs.

\section{Summary and Conclusions}

[29] Based on the climatology of GPS phase scintillation at high latitudes that showed the scintillation predominantly occurring in the ionospheric footprint of the cusp, a probabilistic forecasting method focusing on the cusp is proposed. The GPS data from two cusp stations in Cambridge Bay and Taloyoak in Canada are used to construct time series of scintillation occurrence that is correlated with solar wind velocity. Using a superposed epoch analysis it is shown that there is a significant increase in phase scintillation and cycle slip occurrence just after the arrival of high-speed solar wind CIRs and ICMEs. The mean scintillation activity in the cusp tapers off a few days later. Cumulative probability distribution functions for the phase scintillation occurrence in the cusp during solar minimum are obtained for days before and after CIR and ICME arrivals. The probability curves can also be specified for low and high (below and above median) values of various solar wind plasma parameters. These initial results are used to demonstrate a technique to forecast phase scintillation occurrence in the cusp relative to stream interface between fast and slow solar wind and/or shocks associated with CIRs and ICMEs. A larger statistical sample is needed to refine the probabilistic forecasting for different seasons, levels of solar activity, solar wind velocity magnitude and the IMF orientation, different scintillation levels, and different magnetic latitudes and local times. The method should be applicable to auroral zone and this will be further investigated as the solar activity increases to solar maximum and the coverage of auroral latitudes is improved in the coming years. More work on the validation of forecasting based on cumulative probabilistic curves of phase scintillation occurrence is required before the method can be adapted and included as a sub-product in space weather prediction procedures.

[30] Acknowledgments. Infrastructure funding for CHAIN was provided by the Canada Foundation for Innovation and the New Brunswick Innovation Foundation. CHAIN operation is conducted in collaboration with the Canadian Space Agency (CSA). The solar wind data were obtained from Goddard Space Flight Center Space Physics Data Facility OMNIWeb (http://omniweb.gsfc.nasa.gov/).

\section{References}

Aarons, J. (1982), Global morphology of ionospheric scintillations, Proc. IEE, 70(4), 360-378, doi:10.1109/PROC.1982.12314.

Aarons, J. (1997), Global positioning system phase fluctuations at auroral latitudes, J. Geophys. Res., 102(A8), 17,219-17,231, doi:10.1029/ 97JA01118.

Aarons, J., B. Lin, M. Mendillo, K. Liou, and M. Codrescu (2000), Global Positioning System phase fluctuations and ultraviolet images from the Polar satellite, J. Geophys. Res., 105, 5201-5213, doi:10.1029/1999JA900409.

Alfonsi, L., L. Spogli, G. De Franceschi, V. Romano, M. Aquino, A. Dodson, and C. N. Mitchell (2011), Bipolar climatology of GPS ionospheric scintillation at solar minimum, Radio Sci., 46, RS0D05, doi:10.1029/2010RS004571.

Anderson, D. N., B. Reinisch, C. Valladares, J. Chau, and O. Veliz (2004), Forecasting the occurrence of ionospheric scintillation activity in the equatorial ionosphere on a day-to-day basis, J. Atmos. Sol. Terr. Phys., 66(17), 1567-1572, doi:10.1016/j.jastp.2004.07.010.

Aquino, M., A. Dodson, G. deFranceschi, L. Alfonsi, V. Romano, J. Monico, H. Marques, and C. Mitchell (2007), Towards forecasting and mitigating ionospheric scintillation effects on GNSS, paper presented at 49th International Symposium ELMAR-2007, Inst. of Electr. and Electron. Eng., Zadar, Croatia, 12-14 Sept.

Badruddin, V. G. (2009), Interplanetary structures and solar wind behaviour during major geomagnetic perturbations, J. Atmos. Sol. Terr. Phys., 71(8-9), 885-896.

Basu, S., E. J. Weber, T. W. Bullett, M. J. Keskinen, E. MacKenzie, P. Doherty, R. Sheehan, H. Kuenzler, P. Ning, and J. Bongiolatti (1998), Characteristics of plasma structuring in the cusp/cleft region at Svalbard, Radio Sci., 33(6), 1885-1899, doi:10.1029/98RS01597.

Basu, S., K. M. Groves, Su. Basu, and P. J. Sultan (2002), Specification and forecasting of scintillations in communication/navigation links: Current status and future plans, J. Atmos. Sol. Terr. Phys., 64(16), 1745-1754, doi:10.1016/S1364-6826(02)00124-4.

Basu, S., Su. Basu, K. M. Groves, E. MacKenzie, M. J. Keskinen, and F. J. Rich (2005), Near-simultaneous plasma structuring in the midlatitude and equatorial ionosphere during magnetic superstorms, Geophys. Res. Lett., 32, L12S05, doi:10.1029/2004GL021678.

Basu, S., Su. Basu, E. MacKenzie, C. Bridgwood, C. E. Valladares, K. M. Groves, and C. Carrano (2010), Specification of the occurrence of equatorial ionospheric scintillations during the main phase of large magnetic storms within solar cycle 23, Radio Sci., 45, RS5009, doi:10.1029/2009RS004343. 
Belcher, J. W., and L. Davis Jr. (1971), Large-amplitude Alfvén waves in the interplanetary medium: 2, J. Geophys. Res., 76, 3534-3563, doi:10.1029/JA076i016p03534.

Borovsky, J. E., and M. H. Denton (2006), Differences between CMEdriven storms and CIR-driven storms, J. Geophys. Res., 111, A07S08, doi:10.1029/2005JA011447.

Borovsky, J. E., and J. T. Steinberg (2006), The "calm before the storm" in CIR/magnetosphere interactions: Occurrence statistics, solar wind statistics, and magnetospheric preconditioning, J. Geophys. Res., 111, A07S10, doi:10.1029/2005JA011397.

Buonsanto, M. J. (1999), Ionospheric storms-A review Space Sci. Rev., 88, 563-601, doi:10.1023/A:1005107532631.

Burlaga, L. F. (1974), Interplanetary stream interfaces, J. Geophys. Res., 79, 3717-3725, doi:10.1029/JA079i025p03717.

Conker, R. S., M. B. El Arini, C. J. Hegarty, and T. Hsiao (2003), Modeling the effects of ionospheric scintillation on GPS/SBAS availability, Radio Sci., 38(1), 1001, doi:10.1029/2000RS002604.

Crane, R. K. (1976), Spectra of ionospheric scintillation, J. Geophys. Res., 81(13), 2041-2050, doi:10.1029/JA081i013p02041.

Dabas, R. S., D. R. Lakshmi, and B. M. Reddy (1989), Effect of geomagnetic disturbances on the VHF nighttime scintillation activity at equatorial and low latitudes, Radio Sci., 24(4), 563-573, doi:10.1029/ RS024i004p00563.

Davis, C. J., J. A. Davies, M. J. Owens, and M. Lockwood (2012), Predicting the arrival of high-speed solar wind streams at Earth using the STEREO Heliospheric Imagers, Space Weather, 10, S02003, doi:10.1029/2011SW000737.

de Abreu, A. J., Y. Sahai, P. R. Fagundes, F. Becker-Guedes, R. de Jesus, F. L. Guarnieri, and V. G. Pillat (2010), Response of the ionospheric $F$ region in the Brazilian sector during the super geomagnetic storm in April 2000 observed by GPS, Adv. Space Res., 45(11), 1322-1329, doi:10.1016/j.asr.2010.02.003.

Decker, D. T., and T. R. Pedersen (2001), Downstream forecasting of ionospheric scintillation based on trajectory modeling of in-situ measurements, Eos Trans. AGU, 82(47), Fall Meet. Suppl., Abstract SA51A-0762.

de La Beaujardière, O., L. Jeong, and the C/NOFS Science Definition Team (2004), C/NOFS: A mission to forecast scintillations, J. Atmos. Sol. Terr. Phys., 66, 1573-1591, doi:10.1016/j.jastp.2004.07.030.

Donovan, E., T. Trondsen, L. Cogger, and B. Jackel (2003), Auroral imaging within the Canadian CANOPUS and NORSTAR projects, Publ. 92, pp. 109-112, Sodankylä Geophys. Obs., Sodankylä, Finland.

Emery, B. A., I. G. Richardson, D. S. Evans, F. J. Rich, and G. R. Wilson (2011), Solar rotational periodicities and the semiannual variation in the solar wind, radiation belt, and aurora, Sol. Phys., 274, 399-425, doi:10.1007/s11207-011-9758-x.

Gosling, J. T., J. R. Asbridge, S. J. Bame, and W. C. Feldman (1978), Solar wind stream interfaces, J. Geophys. Res., 83, 1401-1412.

Hegarty, C., M. B. El-Arini, T. Kim, and S. Ericson (2001), Scintillation modeling of GPS wide area augmentation system receivers, Radio Sci., 36, 1221-1231, doi:10.1029/1999RS002425.

Holzworth, R. H., and C.-I. Meng (1975), Mathematical representation of the auroral oval, Geophys. Res. Lett., 2(9), 377-380, doi:10.1029/ GL002i009p00377.

Horvath, I., and S. Crozier (2007), Software developed for obtaining GPS-derived total electron content values, Radio Sci., 42, RS2002, doi:10.1029/2006RS003452.

Insley, J. E., V. Moore, and R. A. Harrison (1995), The differential rotation of the corona as indicated by coronal holes, Sol. Phys., 160, 1-18, doi:10.1007/BF00679089.

Jayachandran, P. T., et al. (2009), Canadian High Arctic Ionospheric Network (CHAIN), Radio Sci., 44, RS0A03, doi:10.1029/ 2008RS004046 [printed 45(1), 2010].

Jian, L., C. T. Russell, J. G. Luhmann, and R. M. Skoug (2006), Properties of stream interactions at one AU during 1995-2004, Sol. Phys., 239(1-2), 337-392, doi:10.1007/s11207-006-0132-3.

Li, G., B. Ning, W. Wan, and B. Zhao (2006), Observations of GPS ionospheric scintillations over Wuhan during geomagnetic storms, Ann. Geophys., 24, 1581-1590, doi:10.5194/angeo-24-1581-2006.

Li, G., B. Ning, B. Zhao, L. Liu, J. Y. Liu, and K. Yumoto (2008), Effects of geomagnetic storm on GPS ionospheric scintillations at Sanya, J. Atmos. Sol. Terr. Phys., 70(7), 1034-1045, doi:10.1016/j.jastp. 2008.01.003.
Liu, W. W. (2005), Canadian space environment program and international living with a star, Adv. Space Res., 35(1), 51-60, doi:10.1016/j. asr.2004.09.011.

Love, J. J., E. Joshua Rigler, and S. E. Gibson (2012), Geomagnetic detection of the sectorial solar magnetic field and the historical peculiarity of minimum 23-24, Geophys. Res. Lett., 39, L04102, doi:10.1029/2011GL050702.

Mannucci, A. J., B. T. Tsurutani, B. A. Iijima, A. Komjathy, A. Saito, W. D. Gonzalez, F. L. Guarnieri, J. U. Kozyra, and R. Skoug (2005), Dayside global ionospheric response to the major interplanetary events of October 29-30, 2003 "Halloween Storms," Geophys. Res. Lett., 32, L12S02, doi:10.1029/2004GL021467.

McPherron, R. L., and G. Siscoe (2004), Probabilistic forecasting of geomagnetic indices using solar wind air mass analysis, Space Weather, 2, S01001, doi:10.1029/2003SW000003.

McPherron, R. L., and J. Weygand (2006), The solar wind and geomagnetic activity as a function of time relative to corotating interaction regions, in Recurrent Magnetic Storms: Corotating Solar Wind Streams, Geophys. Monogr. Ser., vol. 167, edited by B. Tsurutani et al., pp. 125-137, AGU, Washington, D. C., doi:10.1029/167GM12.

Mitchell, C. N., L. Alfonsi, G. De Franceschi, M. Lester, V. Romano, and A. W. Wernik (2005), GPS TEC and scintillation measurements from the polar ionosphere during the October 2003 storm, Geophys. Res. Lett., 32, L12S03, doi:10.1029/2004GL021644.

Miyoshi, Y., and R. Kataoka (2008), Flux enhancement of the outer radiation belt electrons after the arrival of stream interaction regions, J. Geophys. Res., 113, A03S09, doi:10.1029/2007JA012506.

Mlynczak, M. G., F. J. Martin-Torres, C. J. Mertensm, B. T. Marshall, R. E. Thompson, J. U. Kozyra, E. E. Remsberg, L. L. Gordley, J. M. Russell III, and T. Woods (2008), Solar-terrestrial coupling evidenced by periodic behavior in geomagnetic indexes and the infrared energy budget of the thermosphere, Geophys. Res. Lett. 35, L05808, doi:10.1029/2007GL032620.

Navarro-Peralta, P., and A. Sanchez-Ibarra (1994), An observational study of coronal hole rotation over the sunspot cycle, Sol. Phys. 153(1-2), 169-178, doi:10.1007/BF00712499.

Odstrcil, D., V. J. Pizzo, and C. N. Arge (2005), Propagation of the 12 May 1997 interplanetary coronal mass ejection in evolving solar wind structures, J. Geophys. Res., 110, A02106, doi:10.1029/ 2004JA010745.

Osherovich, V. A., J. Fainberg, and R. G. Stone (1999), Solar wind quasi-invariant as a new index of solar activity, Geophys. Res. Lett. 26(16), 2597-2600, doi:10.1029/1999GL900583.

Pedatella, N. M., and J. M. Forbes (2011), Electrodynamic response of the ionosphere to high-speed solar wind streams, J. Geophys. Res., 116, A12310, doi:10.1029/2011JA017050.

Pizzo, V. (1978), A three-dimensional model of corotating streams in the solar wind: 1. Theoretical foundations, J. Geophys. Res., 83, 5563-5572, doi:10.1029/JA083iA12p05563.

Pokhotelov, D., P. T. Jayachandran, C. N. Mitchell, and M. H. Denton (2010), High-latitude ionospheric response to co-rotating interaction region- and coronal mass ejection-driven geomagnetic storms revealed by GPS tomography and ionosondes, Proc. R. Soc. A, 466, 3391-3408.

Prikryl, P., V. Rušin, and M. Rybanský (2009a), The influence of solar wind on extratropical cyclones-Part 1: Wilcox effect revisited, Ann Geophys., 27, 1-30, doi:10.5194/angeo-27-1-2009.

Prikryl, P., D. B. Muldrew, and G. J. Sofko (2009b), The influence of solar wind on extratropical cyclones-Part 2: A link mediated by auroral atmospheric gravity waves?, Ann. Geophys., 27, 31-57, doi:10.5194/angeo-27-31-2009.

Prikryl, P., P. T. Jayachandran, S. C. Mushini, D. Pokhotelov, J. W. MacDougall, E. Donovan, E. Spanswick, and J.-P. St.-Maurice (2010), GPS TEC, scintillation and cycle slips observed at high latitudes during solar minimum, Ann. Geophys., 28(6), 1307-1316, doi:10.5194/angeo-28-1307-2010.

Prikryl, P., P. T. Jayachandran, S. C. Mushini, and R. Chadwick (2011a), Climatology of GPS phase scintillation and HF radar backscatter for the high-latitude ionosphere under solar minimum conditions, Ann. Geophys., 29, 377-392, doi:10.5194/angeo-29-377-2011.

Prikryl, P., P. T. Jayachandran, S. C. Mushini, and R. Chadwick (2011b), GPS phase scintillation and HF radar backscatter occurrence in the high-latitude ionosphere, paper presented at XXX 
General Assembly and Scientific Symposium, Int. Union of Radio Sci., Istanbul, Turkey, 13-20 Aug.

Prikryl, P., et al. (2011c), Interhemispheric comparison of GPS phase scintillation at high latitudes during the magnetic-cloud-induced geomagnetic storm of 5-7 April 2010, Ann. Geophys., 29, 2287-2304, doi:10.5194/angeo-29-2287-2011.

Ray, S., and A. DasGupta (2007), Geostationary L-band signal scintillation observations near the crest of equatorial anomaly in the Indian zone, J. Atmos. Sol. Terr. Phys., 69(4-5), 500-514, doi:10.1016/ j.jastp.2006.09.007.

Redmon, R. J., D. Anderson, R. Caton, and T. Bullett (2010), A Forecasting Ionospheric Real-time Scintillation Tool (FIRST), Space Weather, 8, S12003, doi:10.1029/2010SW000582.

Richardson, I. G., and H. V. Cane (2010), Near-Earth interplanetary coronal mass ejections during solar cycle 23(1996-2009), catalog and summary of properties, Sol. Phys., 264(1), 189-237, doi:10.1007/ s11207-010-9568-6.

Richardson, I. G., et al. (2006), Major geomagnetic storms (Dst $\leq-100$ nT) generated by corotating interaction regions, J. Geophys. Res., 111, A07S09, doi:10.1029/2005JA011476.

Russell, C. T., and R. L. McPherron (1973), Semiannual variation of geomagnetic activity, J. Geophys. Res., 78(1), 92-108, doi:10.1029/ JA078i001p00092.

Secan, J. A., R. M. Bussey, E. J. Fremouw, and S. Basu (1995), An improved model of equatorial scintillation, Radio Sci., 30(3), 607-617, doi:10.1029/94RS03172.

Secan, J. A., R. M. Bussey, E. J. Fremouw, and S. Basu (1997), Highlatitude upgrade to the Wideband ionospheric scintillation model, Radio Sci., 32(4), 1567-1574, doi:10.1029/97RS00453.

Shnirman, M. G., J.-L. Le Mouël, and E. M. Blanter (2010), Slow and fast rotating coronal holes from geomagnetic indices, Sol. Phys., 266, 159-171, doi:10.1007/s11207-010-9605-5.

Skone, S., and M. de Jong (2000), The impact of geomagnetic substorms on GPS receiver performance, Earth Planets Space, 52, 1067-1071.

Sojka, J. J., R. W. Schunk, M. Nicholls, and C. J. Heinselman (2010), Possibility and demonstrations of 27 day ionospheric forecasting, Abstract SM51A-1773 presented at 2010 Fall Meeting, AGU, San Francisco, Calif., 13-17 Dec.

Spogli, L., L. Alfonsi, G. De Franceschi, V. Romano, M. H. O. Aquino, and A. Dodson (2009), Climatology of GPS ionospheric scintillations over high and mid-latitude European regions, Ann. Geophys., 27, 3429-3437, doi:10.5194/angeo-27-3429-2009.

Sreeja, V., M. Aquino, and Z. G. Elmas (2011), Impact of ionospheric scintillation on GNSS receiver tracking performance over Latin America: Introducing the concept of tracking jitter variance maps, Space Weather, 9, S10002, doi:10.1029/2011SW000707.
Strangeways, H. J., Y.-H. Ho, M. H. O. Aquino, Z. G. Elmas, H. A. Marques, J. F. G. Monico, and H. A. Silva (2011), On determining spectral parameters, tracking jitter, and GPS positioning improvement by scintillation mitigation, Radio Sci., 46, RS0D15, doi:10.1029/2010RS004575.

Thayer, J. P., J. Lei, J. M. Forbes, E. K. Sutton, and R. S. Nerem (2008) Thermospheric density oscillations due to periodic solar wind highspeed streams, J. Geophys. Res., 113, A06307, doi:10.1029/2008JA013190.

Tsurutani, B. T., and W. D. Gonzalez (1997), The interplanetary causes of magnetic storms: A review, in Magnetic Storms, Geophys. Monogr. Ser., vol. 98, edited by B. T. Tsurutani et al., pp. 77-89, AGU, Washington, D. C., doi:10.1029/GM098p0077.

Tsurutani, B. T., et al. (2006), Corotating solar wind streams and recurrent geomagnetic activity: A review, J. Geophys. Res., 111, A07S01, doi:10.1029/2005JA011273.

Tsurutani, B. T., E. Echer, and W. D. Gonzalez (2011), The solar and interplanetary causes of the recent minimum in geomagnetic activity (MGA23), A combination of midlatitude small coronal holes, low IMF $B_{z}$ variances, low solar wind speeds and low solar magnetic fields, Ann. Geophys., 29, 1-11, doi:10.519/ angeo-29-839-2011.

Van Dierendonck, A. J., and B. Arbesser-Rastburg (2004), Measuring ionospheric scintillation in the equatorial region over Africa, including measurements from SBAS geostationary satellite signals, paper presented at 17th International Technical Meeting, Satell. Div., Inst. of Navig., Long Beach, Calif.

Verkhoglyadova, O. P., B. T. Tsurutani, A. J. Mannucci, M. G. Mlynczak, L. A. Hunt, A. Komjathy, and T. Runge (2011), Ionospheric VTEC and thermospheric infrared emission dynamics during corotating interaction region and high-speed stream intervals at solar minimum: 25 March to 26 April 2008, J. Geophys. Res., 116, A09325, doi:10.1029/2011JA016604.

Wernik, A. W., L. Alfonsi, and M. Materassi (2007), Scintillation modeling using in situ data, Radio Sci., 42, RS1002, doi:10.1029/ 2006RS003512.

Williams, A. O., N. J. T. Edberg, S. E. Milan, M. Lester, M. Fränz, and J. A. Davies (2011), Tracking corotating interaction regions from the Sun through to the orbit of Mars using ACE, MEX, VEX, and STEREO, J. Geophys. Res., 116, A08103, doi:10.1029/2010JA015719.

Wu, C.-C., M. Dryer, S. T. Wu, B. E. Wood, C. D. Fry, K. Liou, and S. Plunkett (2011), Global three-dimensional simulation of the interplanetary evolution of the observed geoeffective coronal mass ejection during the epoch 1-4 August 2010, J. Geophys. Res., 116, A12103, doi:10.1029/2011JA016947.

Zurbuchen, T. H., and I. G. Richardson (2006), In-situ solar wind and field signatures of interplanetary coronal mass ejections, Space Sci. Rev., 123, 31-43, doi:10.1007/s11214-006-9010-4. 